\title{
A Pathologic Perspective of COVID-19: Parallels with Severe Influenza Virus Pneumonia.
}

Teluguakula Narasaraju ${ }^{1}$, Sunil More ${ }^{1}$, Marko Radic ${ }^{2}$ and Vincent TK Chow ${ }^{3}$.

${ }^{1}$ College of Veterinary Medicine, Oklahoma State University, Stillwater, Oklahoma USA.

${ }^{2}$ Department of Microbiology, Immunology and Biochemistry, College of Medicine, University of Tennessee Health Science Center, Memphis, TN, USA.

${ }^{3}$ Department of Microbiology and Immunology, School of Medicine, National University of Singapore, National University Health System, Singapore.

*Corresponding author: Teluguakula Narasaraju (narasa@okstate.edu)

KEY WORDS: COVID-19, SARS-CoV-2, ARDS, neutrophils, neutrophilia, NETs, pathogenesis 


\section{Acknowledgments:}

This work was supported by the National Institute of General Medical Sciences of the National Institutes of Health (NIH) under Award Number P20GM103648, a grant from the Oklahoma Center for the Advancement of Science \& Technology (OCAST) and a grant from the Center for Veterinary Health Sciences, Oklahoma State University to TN.

\section{Contributions:}

TN performed experiments and drafted the manuscript. SM provided pathology analysis. MR and VC provided key insights for the manuscript. All authors reviewed the final version of the text.

\section{Statement:}

All authors declare that they did not have any conflict of interest in preparing the manuscript. 


\begin{abstract}
Severely ill patients with of the novel coronavirus disease 2019 (COVID-19) experience pathologic manifestations of acute respiratory distress syndrome (ARDS). Although clinical characteristics and lung CT analysis help in determining severity of illness, progressive pathologic changes that occur during the course of infection are not completely understood. Studies have shown high overlapping pathophysiology between severe influenza pneumonia and COVID-19 infections. Here, we attempted to recapitulate how lung pathologic lesions evolve during progressive ARDS complications using a murine fatal influenza model. Based on these studies, we propose three pathologic stages that represent different stages of clinical illness in COVID-19 infections.
\end{abstract}




\section{Introduction.}

The pandemic of coronavirus disease 2019 (COVID-19) is causing devastating numbers of deaths around the world. ${ }^{1-2}$ The majority of patients with COVID-19 recover with mild to moderate symptoms. However, about 10-15\% of patients develop progressive pneumonia with severe to critical pulmonary lesions, and a significant number of these patients succumb to infection with pathologic manifestations of acute respiratory distress syndrome (ARDS). ${ }^{3-4}$ The current management of COVID19 relies mainly on clinical characteristics and lung CT imaging that provide the basis for determining severity of illness and requirement for ventilatory support. ${ }^{5-6}$ Although lung CT analysis can reveal consolidation and bilateral ground-glass opacities, the progressive pathologic changes occurring at different stages of COVID-19 are poorly defined. A clear understanding of pathology at different stages of infection can facilitate the development of new therapeutic strategies to prevent or minimize deaths among critically ill patients with COVID-19.

Currently, there are only a limited number of lung biopsy and autopsy studies that describe the pathology of COVID-19. The gross pulmonary findings mainly revealed highly edematous lungs with focal or diffuse congestion, with variable degrees of hemorrhagic and necrotic lesions. ${ }^{7-10}$ The major lung microscopic lesions observed include diffuse alveolar damage (DAD), widespread hemorrhagic effusions, inflammatory exudation, epithelial denudation, coagulopathy, and abnormal alveolar type II epithelial and fibroblast proliferation. ${ }^{7-8}$ There are also reports that SARS-CoV-2 can affect other organs, including the gastrointestinal tract, liver, heart and kidneys. ${ }^{11-13}$ A report of four patient autopsies showed extensive DAD with degenerative neutrophils filling the bronchioles and alveoli. ${ }^{9}$ Another pulmonary pathology report of postmortem needle core biopsies documented abnormal remodeling with alveolar type II hyperplasia, and proliferation of fibroblasts associated with widespread formation of hyaline membrane, and hemorrhagic exudation. ${ }^{11}$ In addition, lung autopsy reports also 
demonstrated severe bronchopneumonia with peri-bronchial cuffing with neutrophils and induction of neutrophil extracellular traps (NETs) in the blood. ${ }^{10,14}$ However, most of these pathology studies were performed in the context of samples collected postmortem, and there is a dearth of data on how lung pathologic lesions evolve during the different stages of COVID-19 illness.

\section{Lung pathology in COVID-19 share strong similarities with severe influenza pneumonia.}

Pathology reports of fatal influenza infections in humans show strong similarities with SARSCoV-2 infection. Avian influenza H5N1-infected human lung autopsies reveal DAD as the most prominent pathologic lesion, which is associated with hemorrhagic exudation, necrotizing bronchioles, alveolitis, neutrophilic and macrophage-predominant inflammation, alveolar type II hyperplasia. ${ }^{15-16}$ Abnormal tissue remodeling with hyperplasia of pneumocytes and fibrotic proliferation are also observed in fatal influenza, thus supporting the notable overlap with COVID-19. ${ }^{16}$ Similar to pathophysiology in humans, studies using murine and non-primate models of influenza can recapitulate the majority of these pathologic lesions. ${ }^{17-19}$

Given the overlapping pathologies between SARS-CoV-2 and influenza at the critical disease stage, we attempted to reconstruct how pathology evolves during the disease progression using a fatal murine influenza infection model. BALB/c mice (6-8 weeks old, Jackson Laboratory) were challenged with lethal doses of influenza virus A/PR/8/34 (H1N1) (2500 tissue culture infective dose 50 or TCID50) via the intranasal route. All mice (protocol \# VM 17-32) studies were approved by the Institutional Animal Care and Use Committee (IACUC) of Oklahoma State University. Infected mice developed clinical signs of piloerection and lethargy starting from 3 days post-infection (dpi), loss of appetite and difficulty in breathing starting from $5 \mathrm{dpi}$, exhibited hunched posture with respiratory distress between 6-8 dpi, and died between 8-9 dpi. Animals were euthanized with overdose of ketamine and xylazine at 
0, 3, 6 and 8 dpi. Whole lungs were fixed by intranasal instillation of neutral-buffered formalin, dehydrated, embedded in wax, and 4- $\mu \mathrm{m}$ thick sections were prepared for lung histopathologic analyses. Lung pathology at different time intervals revealed two distinct pathologic signatures. At 3 dpi, infected lungs exhibited widespread inflammation, but minimal hemorrhagic lesions (Figure 1). Lungs at $3 \mathrm{dpi}$ prominently displayed severe necrotizing bronchiolitis, epithelial denudation, extensive neutrophil influx, alveolar injury, and interstitial inflammation similar to the pathologic lesions found in patients infected with COVID-19. ${ }^{7,9}$ By 6 dpi, animals developed pathologic lesions of DAD with prominent hemorrhagic lesions and proliferating bronchiolar epithelial cells were observed. By 8 dpi, the lungs displayed widespread DAD with parenchymal hemorrhage and vascular leakage. Areas of lungs with severe pathology displayed widespread pulmonary edema, vascular disruption, and fibrin accumulation in the alveoli. The bronchiolar epithelium demonstrated multifocal necrosis and sloughing. In parallel to these pathology, analyses of bronchoalveolar lavage (BAL) identified markers of epithelial and endothelial damage, accumulation of extracellular histones and toxic granule proteins, which contribute

to ARDS pathology. ${ }^{18-19}$ In addition, increased vascular thrombosis and plasma fibrinogen degradation products also found in these mice ${ }^{18}$ thus recapitulates pathologic lesions of COVID-19 infection.

Based on the available pathologic data, clinical features, and laboratory findings, we can reasonably categorize pathologic lesions of COVID-19 into three stages from the onset of the illness, i.e. mild, severe and critical, as outlined in Figure 2.

\section{Stage 1: Early mild infection.}

During the early stage, the virus invades the upper respiratory tract, and initiates spreading deeper into the lungs. SARS-CoV-2 binds to angiotensin-converting enzyme 2 (ACE2) receptors on epithelial cells of respiratory epithelium and immune cells such as macrophages. This stage of virus 
infection can cause cytopathic effects, and induce cytokine and chemokine responses, which eventually trigger inflammatory responses. Although the cellular tropism of SARS-CoV-2 is yet to be fully elucidated, the virus can infect bronchiolar ciliated epithelium, goblet cells, alveolar type I and type II pneumocytes, macrophages, and dendritic cells. ${ }^{20}$ Importantly, infected patients at this stage may serve as potential sources for transmission of the virus.

\section{Stage 2: Severe clinical illness.}

As the virus migrates into the deeper lungs and aggravates cytopathic effects, it also causes extensive influx of immune cells. This second phase can be defined as the inflammatory exudative stage during which massive numbers of neutrophils, macrophages and histiocytes are recruited into the infected lungs. These lesions have been reported in both lung biopsies and autopsies from COVID-19 patients. The activated neutrophils likely instigate acute alveolar-capillary damage by generating toxic granule proteins (such as neutrophil elastase and matrix metalloproteinases including MMP-9 and MMP-2) and components of NETs such as extracellular histones (ECH). The released proteases can disrupt extracellular matrix protein in the basement membrane, causing epithelial sloughing. Further, degradation of basement membrane also exposes the underlying pulmonary vasculature, which is targeted by ECH to induce vascular thrombosis and these pathologic lesions are most predominantly described in lung autopsies from COVID-19 patients. ${ }^{79}$ We previously reported that injured epithelial cells during influenza pneumonia are regenerated by growth factors such as hepatocyte growth factor, produced by myeloid lineage cells simultaneously. ${ }^{21}$ Thus, it is likely that epithelial hyperplasia and fibroblast proliferation begin during this stage, and become more obvious at the critical stage of infection. During this phase, the lung CT scan would likely appear as focal ground-glass opacities, and may be regarded as the "early stage" of ARDS pathophysiology. 


\section{Stage 3: Critically ill phase}

COVID-19 patients who develop critical illness likely display progressive manifestations of ARDS. The lung autopsies or postmortem biopsies from these patients show widespread hemorrhagic effusions evident by alveoli filled with red blood cells and cellular debris. Extensive DAD with pulmonary edema, fibrotic proliferation, hyaline membrane formation are reflected by impaired gas exchange and these patients succumb to infection with respiratory failure despite ventilation therapy. ${ }^{22}$ Furthermore, heightened levels of D-dimers indicate pro-thrombotic status in the pulmonary vasculature. We believe that these severe pathologic lesions likely arise from cumulative effects of prior inflammation, epithelial necrosis, and protease-mediated ECM degradation and vascular injury. Design of additional diagnostic methods using less invasive approaches such as nasal wash or mini-BAL analysis to assess acute lung injury, ${ }^{23}$ in addition to the current lung CT analysis will likely help clinicians in better understanding the pathophysiology of the disease at different stages.

In conclusion, we propose that lessons learnt from severe influenza pneumonia models can provide pertinent insights into the progression of key pathologic events during the evolution of COVID19 illness. Developing therapeutic strategies that target crucial pathologic events at the severe phase, especially targeting epithelial loss and blocking protease-mediated alveolar-capillary damage will likely prevent or ameliorate the progressive pathologic complications of ARDS, thus effectively mitigating morbidity and mortality in severely ill COVID-19 patients. 


\section{References:}

1. Wu JT, Leung K, Leung GM. Now casting and forecasting the potential domestic and international spread of the 2019-nCoV outbreak originating in Wuhan, China: a modelling study. Lancet. 2020; 395: 689-697

2. Zhu N, Zhang D, Wang W, Li X, Yang B, Song J, Zhao X, Huang B, Shi W, Lu R, Niu P, Zhan F, Ma X, Wang D, Xu W, Wu G, Gao GF, Tan W; A Novel coronavirus from patients with pneumonia in China, 2019. N Engl J Med. 2020. doi: 0.1056/NEJMoa200101

3. Li X, Ma X. Acute respiratory failure in COVID-19: is it "typical" ARDS? Crit Care. 2020 May 6;24(1):198. doi: 10.1186/s13054-020-02911-9.

4. Pan C, Chen L, Lu C, Zhang W, Xia J, Sklar MC, Du M, Brochard L, Qiu H, Lung Recruitability in SARS-CoV-2 Associated Acute Respiratory Distress Syndrome: A Single-center, Observational Study. Am J Resp Crit Care Med. 2020. DOI: 10.21203/rs.3.rs-16485/v1

5. Wu C, Chen X, Cai Y, Xia J, Zhou X, Xu S, Huang H, Zhang L, Zhou X, Du C, Zhang Y, Song J, Wang S, Chao Y, Yang Z, Xu J, Zhou X, Chen D, Xiong W, Xu L, Zhou F, Jiang J, Bai C, Zheng J, Song Y. Risk Factors Associated With Acute Respiratory Distress Syndrome and Death in Patients With Coronavirus Disease 2019 Pneumonia in Wuhan, China. JAMA Intern Med. 2020. doi:10.1001/jamainternmed.2020.0994

6. Wang Y, Dong C, Hu Y, Li C, Ren Q, Zhang X, Shi H, and Zhou M. Temporal Changes of CT Findings in 90 Patients with COVID-19 Pneumonia: A Longitudinal Study. Radiology. March 2020:200843. doi:10.1148/radiol.2020200843.

7. Zhang H, Zhou P, Wei Y, Yue H, Wang Y, Hu M, Zhang S, Cao T, Yang C, Li M, Guo G, Chen X, Chen Y, Lei M, Liu H, Zhao J, Peng P, Wang CY, Du R. Histopathologic Changes and 
SARS-CoV-2 Immunostaining in the Lung of a Patient With COVID-19. Ann Intern Med. 2020:M20-0533. doi: 10.7326/M20-0533.

8. Barton LM, Duval EJ, Stroberg E, Ghosh S, Mukhopadhyay S. COVID-19 Autopsies, Oklahoma, USA. Am J Clin Pathol. 2020 May 5;153(6):725-733. doi: 10.1093/ajcp/aqaa062.

9. Tian S, Xiong Y, Liu H, Niu L, Guo J, Lio M, Xiao Y. Pathological study of the 2019 novel coronavirus disease (COVID-19) through postmortem core biopsies. Mod Pathol. 2020. https://doi.org/10.1038/s41379-020-0536

10. Barnes BJ, Adrover JM, Baxter-Stoltzfus A, Borczuk A, Cools-Lartigue J, Crawford JM, DaßlerPlenker J, Guerci P, Huynh C, Knight JS, Loda M, Looney MR, McAllister F, Rayes R, Renaud S, Rousseau S, Salvatore S, Schwartz RE, Spicer JD, Yost CC, Weber A, Zuo Y, Egeblad M. Targeting potential drivers of COVID-19: Neutrophil extracellular traps. J Exp Med. 2020 Jun 1;217(6):e20200652.

11. Fox SE, Akmatbekov A, Harbert JL, Li G, Brown JQ, Vander Heide RS. Pulmonary and Cardiac Pathology in Covid-19: The First Autopsy Series from New Orleans. MedRxiv 2020.04.06.20050575; doi: ttps://doi.org/10.1101/2020.04.06.20050575

12. Feng G, Zheng KI, Yan QQ, Rios RS, Targher G, Byrne CD, Poucke SV, Liu WY, Zheng MH. COVID-19 and Liver Dysfunction: Current Insights and Emergent Therapeutic Strategies. J Clin Transl Hepatol. 2020 Mar 28;8(1):18-24. doi: 10.14218/JCTH.2020.00018.

13. Divani AA, Andalib S, Napoli MD, Lattanzi S, Hussain MS, Biller J, McCullough JD, Azarpazhooh MR, Seletska A, Mayer SA, Torbey M. Coronavirus Disease 2019 and Stroke: Clinical Manifestations and Pathophysiological Insights. J Stroke Cerebrovasc Dis. 2020; 104941. doi: 10.1016/j.jstrokecerebrovasdis.2020.104941 
14. Zuo Y, Yalavarthi S, Shi H, Gockman K, Zuo M, Madison JA, Blair C, Weber A, Barnes BJ, Egeblad M, Woods RJ, Kanthi Y, Knight JS. Neutrophil extracellular traps (NETs) as markers of disease severity in COVID-19. J Clin Invest insight. 2020 Apr 24. pii: 138999.doi.org/10.1101/2020.04.09.20059626.

15. Ng WF, To KF, Lam WW, Ng TK, Lee KC. The comparative pathology of severe acute respiratory syndrome and avian influenza A subtype H5N1--a review. Hum Pathol. 2006;37:38190.

16. van den Brand JM, Haagmans BL, van Riel D, Osterhaus AD, Kuiken T. The pathology and pathogenesis of experimental severe acute respiratory syndrome and influenza in animal models. J Comp Pathol. 2014;151:83-112.

17. Watanabe T, Iwatsuki-Horimoto K, Kiso M, Nakajima N, Takahashi K, Jose da Silva Lopes T, Ito M, Fukuyama S, Hasegawa H and Kawaoka Y. Experimental infection of Cynomolgus Macaques with highly pathogenic H5N1 influenza virus through the aerosol route. Sci Rep. $2018 ; 8,4801$.

18. Ashar HK, Mueller NC, Rudd JM, Snider TA, Achanta M, Prasanthi M, Pulavendran S, Thomas PG, Ramachandran A, Malayer JR, Ritchey JW, Rajasekhar R, Chow VTK, Esmon CT, Narasaraju T. Role of extracellular histones in influenza virus pathogenesis. Am J Pathol. 2018; 188: $135-148$.

19. Narasaraju T, Edwin Y, Ramar perumal S, Ng HH, Poh WP, Audrey-Ann L, Phoon Meng C, van Rooijen N and Chow VT. Excessive Neutrophils and Neutrophil Extracellular Traps Contribute to Acute Lung Injury of Influenza Pneumonitis. Am J Pathol. 2011; 179: 199-210. 
20. Narasaraju T, Ng HH, Phoon MC, Chow VT. MCP-1 antibody treatment enhances damage and impedes repair of the alveolar epithelium in influenza pneumonitis. Am J Respir Cell Mol Biol. 2010;42:732-43.

21. To KF, Tong JH, Chan PK, Au FW, Chim SS, Chan KC, Cheung JL, Liu EY, Tse GM, Lo AW, Lo YM, Ng HK. Tissue and cellular tropism of the coronavirus associated with severe acute respiratory syndrome: an in-situ hybridization study of fatal cases. J Pathol. 2004;202:157-63.

22. Richardson S, Hirsch JS, Narasimhan M, Crawford JM, McGinn T, Davidson KW; and the Northwell COVID-19 Research Consortium, Barnaby DP, Becker LB, Chelico JD, Cohen SL, Cookingham J, Coppa K, Diefenbach MA, Dominello AJ, Duer-Hefele J, Falzon L, Gitlin J, Hajizadeh N, Harvin TG, Hirschwerk DA, Kim EJ, Kozel ZM, Marrast LM, Mogavero JN, Osorio GA, Qiu M, Zanos TP. Presenting Characteristics, Comorbidities, and Outcomes Among 5700 Patients Hospitalized With COVID-19 in the New York City Area. JAMA. 2020 Apr 22:e206775. doi: 10.1001/jama.2020.6775.

23. Tasbakan MS, Gurgun A, Basoglu OK, Ekren PK, Pullukcu H, Bacakoglu F. Comparison of bronchoalveolar lavage and mini-bronchoalveolar lavage in the diagnosis of pneumonia in immunocompromised patients. Respiration. 2011;81:229-235. 
Figure 1. Temporal changes in lung histopathology and BAL inflammatory cellular signature in the lethal influenza virus infection model of BALB/c mice. Temporal dynamics of hematoxylin-andeosin-stained lung sections or broncho-alveolar lavage (BAL) cells at 3 dpi, 6 dpi and 8 dpi collected from mice challenged with lethal dose of influenza virus versus control mock-infected mice. Lung pathologic lesions progress from inflammatory phase to exudative hemorrhagic changes between 3, 6 and 8 dpi. Epithelial denudation (arrow) and inflammation (asterisk) were prominently observed at 3

dpi. By 6 dpi, infected lungs exhibited focal hemorrhage, tissue consolidation, DAD, and regeneration of epithelium (arrow head). At 8 dpi, infected lungs displayed widespread hemorrhage (square) and hyaline membrane formation (open arrow). Hyperproliferation of alveolar type II pneumocytes (circle) was also observed, together with increased thickening of alveoli in damaged areas of the lungs. Accordingly, the BAL cell signatures depicted these temporal pathologic alterations during the course of infection from inflammatory phase to hemorrhagic exudative phase, i.e. increased neutrophil-predominant influx at 3 dpi, with significantly greater presence of erythrocytes between 3 to 8 dpi. The lungs of mock-infected animals contained mainly alveolar macrophages, with normal lung architecture. $\mathrm{M}=$ macrophage; $\mathrm{L}=$ lymphocyte; $\mathrm{N}=$ neutrophil; $\mathrm{R}=$ red blood cell; $\mathrm{EP}=$ epithelial cell. Shown are representative images of 5 mice per group.

Figure 2. Proposed model of changes in pathologic signatures at different stages of progression of COVID-19. 
Figure 1.

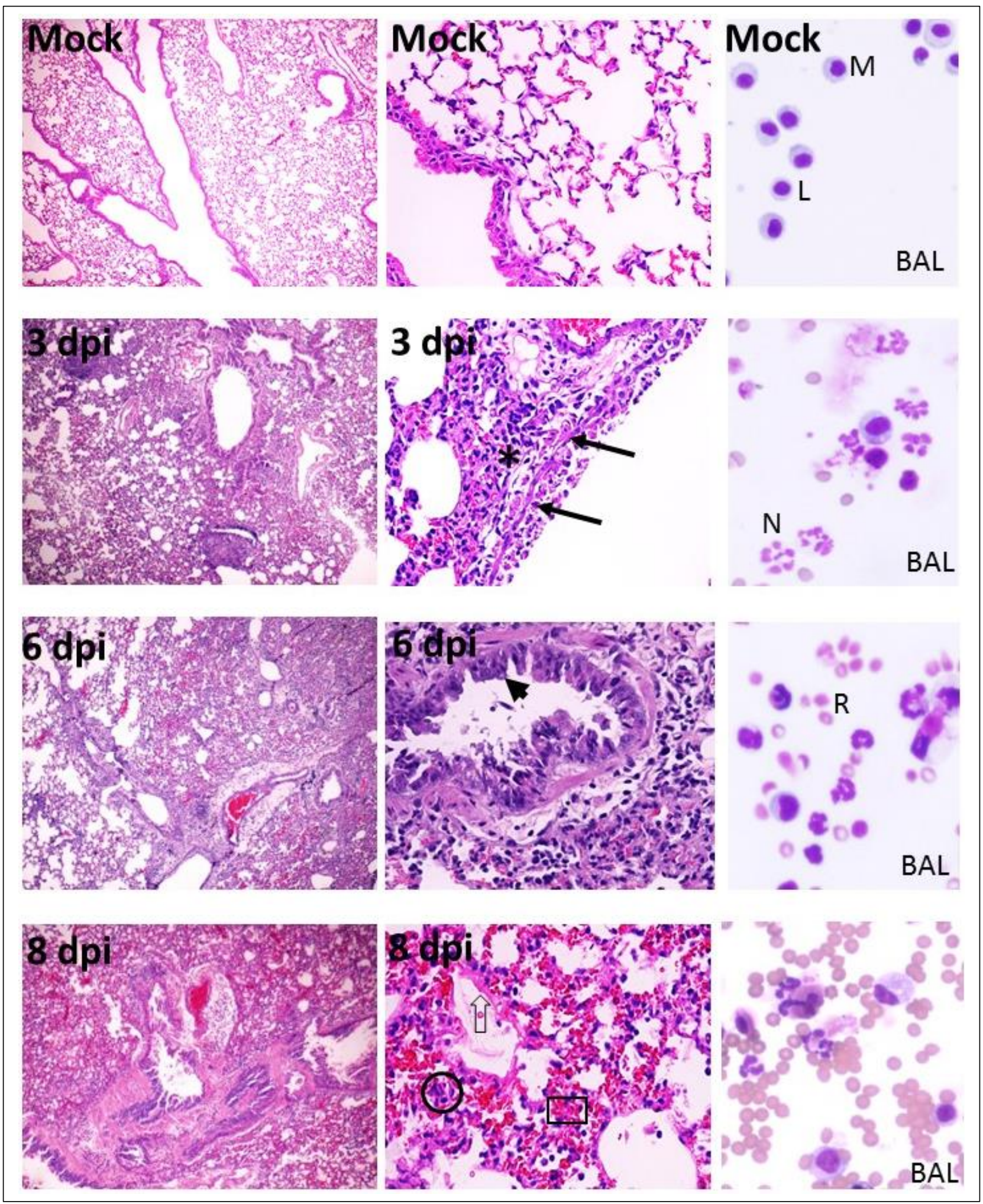


Figure 2.

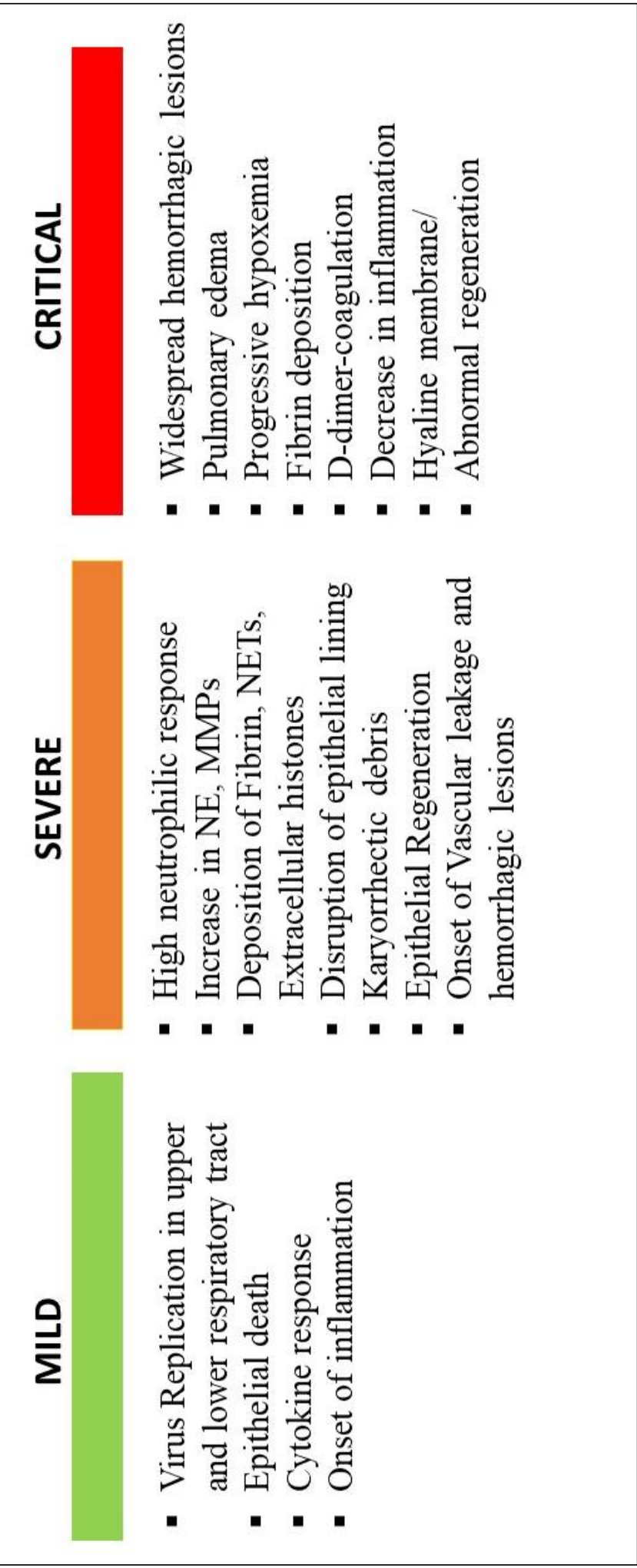

